\title{
Covalent triazine framework with efficient photocatalytic activity in aqueous and solid media
}

\author{
Cyrine Ayed $^{1,2}$, Wei Huang ${ }^{1}$, Kai A. I. Zhang $(\bowtie)^{1}$ \\ 1 Max Planck Institute for Polymer Research, 55128 Mainz, Germany \\ 2 Graduate School for Excellence Materials Science in Mainz, Johannes Gutenberg University Mainz, 55128 Mainz, Germany
}

(C) The Author(s) 2020. This article is published with open access at link.springer.com and journal.hep.com.cn 2020

\begin{abstract}
Covalent triazine frameworks (CTFs) have been recently employed for visible light-driven photocatalysis due to their unique optical and electronic properties. However, the usually highly hydrophobic nature of CTFs, which originates from their overall aromatic backbone, leads to limitations of CTFs for applications in aqueous media. In this study, we aim to extend the range of the application media of CTFs and design hybrid material of a CTF and mesoporous silica (SBA-15) for efficient photocatalysis in aqueous medium. A thiophene-containing CTF was directly synthesized in mesopores of SBA-15. Due to the high surface area and the added hydrophilic properties by silica, the hybrid material demonstrated excellent adsorption of organic molecules in water. This leads not only to high photocatalytic performance of the hybrid material for the degradation of organic dyes in water, but also for efficient photocatalysis in solvent-free and solid state. Furthermore, the reusability, stability and easy recovery of the hybrid material offers promising metal-free heterogeneous photocatalyst for broader applications in different reaction media.
\end{abstract}

Keywords photocatalysis, covalent triazine framework, aqueous medium, SBA-15, solid state

\section{Introduction}

Covalent triazine frameworks (CTFs) represent an interesting and novel class of porous organic polymers, which have considerable unique properties. In particular, the strong aromatic covalent bonds and the rich nitrogen content in the triazine unit endow CTFs with high chemical and thermal stability and desired electronic and optical

Received March 29, 2019; accepted July 3, 2019

E-mail: kai.zhang@mpip-mainz.mpg.de properties [1-3]. These materials can endure harsh conditions such as acid or basic conditions. Moreover, the structure and functionalities of CTFs can be easily adjusted by careful selection of the monomers and corresponding building blocks, which offers an excellent tunability of their physical and chemical properties. From a synthetic view, CTFs can be easily constructed via a vast number of reaction routes by choosing readily available starting materials. All these special properties make CTFs attractive for various applications, such as separation and storage of gases [4,5], energy storage [6-8], photocatalysis [9-12] and heterogeneous catalysis $[13,14]$.

Nevertheless, the impact of the adsorption properties of organic molecules on CTFs during the application process is not well understood yet. The adsorptive power is of keyimportance, particularly in the field of heterogeneous catalysis, where the catalytic reactions occurs at the surface rather than in the solution. Therefore, the surface properties of CTFs become critical for their catalytic efficiencies. In this regard, few studies were interested in investigating closely the adsorptive behavior of CTFs to organic compounds or pollutants. It has been found that these properties [11-13] depends partly on the surface chemistry of adsorbents $[15,16]$. This statement was further confirmed by Kuhn et al. [17]. The authors have found out that good adsorption properties should be ideally achieved with a structure that have extremely high surface area, well defined porosity and good accessibility throughout the pore channel of the functional materials. Thus, the development of porous systems with well-defined morphology is highly beneficial for promoting the adsorption and diffusion of the target molecules and therefore enhancing the performance of the materials, here in particular of this study, the catalysis.

Herein, we report the design of a hybrid photocatalytic system consisting of a thiophene-containing CTF (CTF$\mathrm{Th}$ ) in a mesoporous silica (SBA-15) for visible-light promoted efficient degradation of organic contaminants in 
aqueous media. The presence of mesoporous silica in the hybrid material (CTF-Th@SBA-15) with an ordered mesoporous structure in SBA-15 made the designed system a highly suitable host matrix with two main benefits: (1) The high surface area and ordered pore structure can enhance the diffusion and adsorption of guest molecules, which can boost the photocatalytic efficiency of the CTF. (2) The added hydrophilicity of silica to the photocatalytic CTF can largely extend its application range from organic to aqueous media. The hybrid material consisting of CTF-Th and SBA-15 demonstrated excellent adsorption of organic molecules in water and excellent photodegradation performance of the dyes. Furthermore, after the quick adsorption of the organic dyes in water, the hybrid material also demonstrated its high photocatalytic activity in dry and solid state. Thus, such system opens up new opportunities for efficient adsorption of organic contaminants from water in a wider range of environmentally relevant applications.

\section{Experimental}

\subsection{Materials}

5-Dibromothiophene, copper cyanide, tetraethylorthosilicate (TEOS), trifluromethanesulfonic acid $(\mathrm{TfOH})$, ferric chloride hexahydrate $\left(\mathrm{FeCl}_{3}, 6 \mathrm{H}_{2} \mathrm{O}\right)$, hydrochloric acid (37\%), copper (II) chloride (97\%), ammonium oxalate (99\%), sodium azide (99\%), rhodamine B $(99 \%)$, orange G and methylene blue $(\geqslant 82 \%)$ and all other solvents were purchased from Sigma-Aldich. All chemicals and solvents were used without further purification.

\subsection{Synthesis of 2,5-dicyanothiophene (DCT)}

2,5-Dicyanothiophene was synthesized according to the reported literature [12]. Typically, 2,5-dibromothiophene $(2.0 \mathrm{~g}, 8.3 \mathrm{mmol}, 1 \mathrm{eq}), \mathrm{CuCN}(2.2 \mathrm{~g}, 24.6 \mathrm{mmol}, 3 \mathrm{eq})$ and $N, N$-dimethylmethanamide $(10 \mathrm{~mL})$ were mixed in a 50 $\mathrm{mL}$ flash and heated at reflux at $140^{\circ} \mathrm{C}$ under an inert atmosphere of nitrogen for $12 \mathrm{~h}$. After cooling down to $60^{\circ}$ $\mathrm{C}, \mathrm{FeCl}_{3} \cdot 6 \mathrm{H}_{2} \mathrm{O}(13 \mathrm{~g})$ in $2 \mathrm{~mol} \cdot \mathrm{L}^{-1} \mathrm{HCl}(30 \mathrm{~mL})$ was added and then mixed vigorously for $4 \mathrm{~h}$. The reaction mixture was then cooled down to room temperature and extracted with dichloromethane $(100 \mathrm{~mL} \times 3)$. The combined organic phase was washed with diluted $\mathrm{HCl}$ and Milli Q water, dried with anhydrous $\mathrm{Na}_{2} \mathrm{SO}_{4}$ and concentrated under vacuum. The crude product was obtained as colorless needles (yield 54\%) after being purified through a silica column using hexane: dichloromethane (1:1) as eluent. ${ }^{13} \mathrm{C}$ NMR $(300 \mathrm{MHz}, \mathrm{CDCl} 3) \delta$ 136. 93, 116.1, 111.91 (Fig. S1, cf. Electronic Supplementary Material (ESM)).

\subsection{Synthesis of mesoporous silica SBA-15}

Silica SBA-15 was synthesized according to the reported literature [12]. Simply, a mixture of 4.0 g pluronic P123, $30 \mathrm{~mL}$ of Mill-Q water and $120 \mathrm{~mL}$ of $2 \mathrm{~mol} \cdot \mathrm{L}^{-1} \mathrm{HCl}$ aqueous solution was stirred at $35^{\circ} \mathrm{C}$ overnight. Then 9.1 $\mathrm{mL}$ of TEOS was slowly added into the solution under vigorous stirring. The mixture was first kept at $35^{\circ} \mathrm{C}$ for $24 \mathrm{~h}$ under static conditions, and then heated to $100^{\circ} \mathrm{C}$ for another $24 \mathrm{~h}$. After collection by centrifugation; the resulting precipitate was washed with water, dried and calcined at $550^{\circ} \mathrm{C}$ in air for $4 \mathrm{~h}$. The product was obtained as white solid powder.

\subsection{Fabrication of CTF-Th@SBA-15}

The CTF-Th@SBA-15 was obtained via TfOH vaporassisted solid phase reaction routine according to our previous work [18]. Typically, $200 \mathrm{mg}$ vacuum-dried silica SBA-15 and $100 \mathrm{mg}$ of DCT were first dispersed in $2 \mathrm{~mL}$ of tetrahydrofuran, then stirred for $2 \mathrm{~h}$ under vacuum. The resulting mixture was concentrated under vacuum to give the monomer casted precursor DCT/SBA-15 and further annealed at $80^{\circ} \mathrm{C}$ for $2 \mathrm{~h}$. The precursor was then transferred into a conical flask, in which there was another vial with $0.3 \mathrm{~mL} \mathrm{TfOH}$. The conical flask was kept under an inert atmosphere of nitrogen, sealed and heated up to $100^{\circ} \mathrm{C}$ in an oil bath for $24 \mathrm{~h}$. After cooling down to room temperature, the product was submerged in alkaline distilled aqueous ammonia ( $\mathrm{pH}=8$ to 9) to remove the residual $\mathrm{TfOH}$. Further purification was conducted by washing the sample with water and small volume of acetone, and then dried at $80^{\circ} \mathrm{C}$ under vacuum overnight. The solid catalyst was obtained as a yellow powder.

\subsection{Characterization}

Ultraviolet-visible (UV-Vis) absorption was performed at room temperature on a Perkin Elmer Lambda 100 spectrophotometer. Fourier Transform Infrared spectra (FTIR) were obtained on a Varian 1000 FTIR spectrometer. Solid-state cross-polarization magic angle spinning carbon-13 nuclear magnetic resonance measurements were carried out using Bruker Avance II solid state NMR spectrometer operating at $300 \mathrm{MHz}$ Larmor frequency equipped with a standard $4 \mathrm{~mm}$ magic angle spinning double resonance probe head. The nitrogen sorption data were measured to determine the surface area and the pore size distribution; the data were recorded at $77.3 \mathrm{~K}$ on an Autosorb 1 using QuadraWin Software (Quantchrome instruments). The samples were degassed at $120^{\circ} \mathrm{C}$ overnight under high vacuum before analysis. High resolution transmission electron microscope (HR-TEM) images were performed on a FEI Tecnai F20 with an EDX detector, whereas Scanning electron microscope (SEM) images 
were acquired on a LEO Gemini 1530 (Carl Zeiss AG), using an in lens SE detector. Cyclic voltammetry (CV) measurement was performed using an Autolab PGSTAT204 potentiostat/galvanostat (Metrohm). Glassy carbon electrode drop-casted with the polymer as the working electrode, $\mathrm{Pt}$ wire as the counter electrode, $\mathrm{Hg}$ / $\mathrm{HgCl}$ (in saturated $\mathrm{KCl}$ solution) electrode as the reference electrode, $\mathrm{Bu}_{4} \mathrm{NPF}_{6}\left(0.1 \mathrm{~mol} \cdot \mathrm{L}^{-1}\right.$ in acetonitrile $)$ was used as electrolyte. The water contact angle measurements were recorded by Data Physics OCA 35 (Optical Contact Angle) device, with water droplet volume of ca. $5 \mu \mathrm{L}$, placed with a motor-driven syringe onto the sample surface.

2.6 General procedure for photocatalytic degradation of organic dyes in aqueous and solid media

The photocatalytic performance of CTF-Th@SBA-15 was first tested for the degradation of Rhodamine $\mathrm{B}(\mathrm{RhB})$ in aqueous solution. A mixture of CTF-Th@SBA-15 photocatalyst dispersed in water $\left(0.3 \mathrm{mg} \cdot \mathrm{mL}^{-1}\right)$ and $\mathrm{RhB}$ solution $\left(50 \mathrm{mg} \cdot \mathrm{L}^{-1}, 10 \mathrm{~mL}\right)$ was stirred under dark condition for $60 \mathrm{~min}$ in order to reach the adsorption/ desorption equilibrium between the catalyst and the solution. Then, the mixture was irradiated with blue LED lamp $\left(\lambda=460 \mathrm{~nm}, 0.16 \mathrm{~W} \cdot \mathrm{cm}^{-2}\right)$ during different time intervals in air. Changes in $\mathrm{RhB}$ concentration were monitored using UV-Vis spectroscopy. A control experiment with the reaction mixture under $\mathrm{N}_{2}$, therefore, without oxygen in the reaction, was also performed. The reaction was also tested using pure CTF-Th (without SBA$15)$ as photocatalyst and under the same conditions. The radical scavenger experiments were performed using ammonium oxalate, copper chloride, sodium azide and catalase, in excess $\left(0.05 \mathrm{mmol} \cdot \mathrm{L}^{-1}\right)$. Each of the radical scavengers was added to a mixture of CTF-Th@SBA-15 ( $3 \mathrm{mg}, 10 \mathrm{~mL}$ ) and $\mathrm{RhB}$ solution $\left(50 \mathrm{mg} \cdot \mathrm{L}^{-1}\right)$.

For the solid-state photodegradation experiment, the procedure is described as below: 25 mg of CTF-Th@SBA15 were added to an aqueous solution of $\mathrm{RhB}\left(50 \mathrm{mg} \cdot \mathrm{L}^{-1}\right.$, $10 \mathrm{~mL}$ ) and vigorously stirred for at least $1 \mathrm{~h}$, until the dye particles were completely adsorbed on the surface of the catalyst. Then, the dispersion was filtrated under vacuum and the resulting solid was simply collected. Afterwards, the dried dye-adsorbed catalyst was casted on a flat surface of tape to form a thin layer of powder. The blue LED lamp was also used continuously in this experiment by placing it $1 \mathrm{~cm}$ ahead from the powder layer. The dye degradation was monitored by the measurement of the UV-Vis spectra after certain time intervals. The reaction is considered as finished when the catalyst reacquired its initial color (yellow), due to the complete degradation of the dye particles. Note that two more organic dyes such as methylene blue (MB) and orange G (OrG) were tested following the same procedure described above.

\section{Results and discussion}

\subsection{Materials synthesis and characterization}

CTF-Th@SBA-15 was synthesized onto mesoporous silica SBA-15 via cyclization polymerization of DCT under TfOH vapor, obtaining CTF-Th@SBA-15 as a type of mesoporous nanoreactor. The synthetic pathway with detailed characterization methods of the materials are described in Section 2. The hybrid photocatalyst was obtained as an insoluble yellow powder with the structure shown below (Fig. 1(a)). HR-TEM revealed two-dimensional (2D) hexagonal channels of the CTF-Th@SBA-15, as displayed in Fig. 1(b). SEM confirmed the hexagonal cylinder morphology with a diameter of ca. $500 \mathrm{~nm}$ (see Fig. 1(c)).

The nitrogen gas sorption isotherms and pore size distributions are displayed in Fig. S2 (cf. ESM). A typical hysteresis at a relative pressure of $0.4<P / P_{0}<0.8$ for mesopores is shown for both pristine of SBA-15 as well as the CTF-Th@SBA-15. The corresponding data are summarized in Table 1. The Brunauer-Emmett-Teller (BET) surface area of CTF-Th@SBA-15 was measured to be $374 \mathrm{~m}^{2} \cdot \mathrm{g}^{-1}$, with a pore volume of $0.59 \mathrm{~cm}^{3} \cdot \mathrm{g}^{-1}$, which is lower than the BET surface area of pristine SBA-15, which is $596 \mathrm{~m}^{2} \cdot \mathrm{g}^{-1}$ with a pore volume of $0.9 \mathrm{~cm}^{3} \cdot \mathrm{g}^{-1}$. Note that CTF-Th@SBA-15 exhibited a narrower pore size distribution at ca. $4.1 \mathrm{~nm}$ comparing to that of the pristine SBA-15 of ca. $5.4 \mathrm{~nm}$. This confirms the formation of layer of CTF-Th of an average thickness of ca. $1.3 \mathrm{~nm}$ in the mesopores of SBA-15. In contrast, pure CTF-Th exhibited very low BET surface area due to the absence of the mesoporous channels, as shown in our previous study [12].

FTIR spectra (Fig. 1(d)) showed two intensive peaks at 1486 and $1341 \mathrm{~cm}^{-1}$, which can be assigned to the aromatic $\mathrm{C}-\mathrm{N}$ stretching and breathing modes in the triazine unit. Figure 1(e) illustrates the UV-Vis DR spectra of the CTFTh@SBA-15. The latter showed a broad absorption up to ca. $570 \mathrm{~nm}$. An optical band gap of $2.39 \mathrm{eV}$ could be calculated. These values showed that the as-prepared CTFTh@SBA-15 is a semiconductor that can be excited by visible light. To gain further insight into the electronic properties, CV measurements were conducted to reveal the energy band structure of the designed material. The electronic band position of the CTF-Th@SBA-15 is displayed in Fig. 1(f). The corresponding highest occupied molecular orbital level at $+1.72 \mathrm{~V}$ vs. SCE could be estimated by subtracting the lowest unoccupied molecular orbital (LUMO) level from the optical bandgap (Fig. S3, cf. ESM), indicating a possible high oxidative power of CTF-Th@SBA-15.

To address the wetting property of the hybrid CTFTh@SBA-15 with respect to water, the contact angle of a water droplet on the corresponding surface has been 

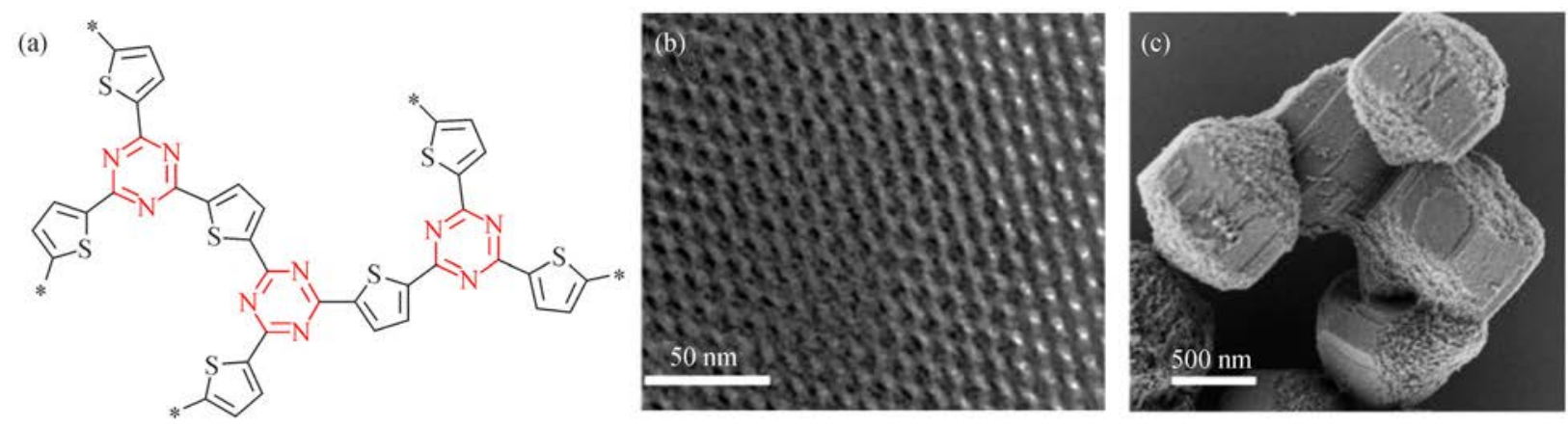

(d)

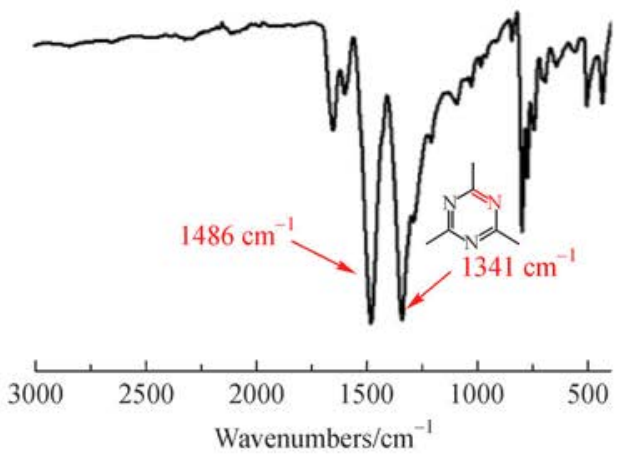

(e)

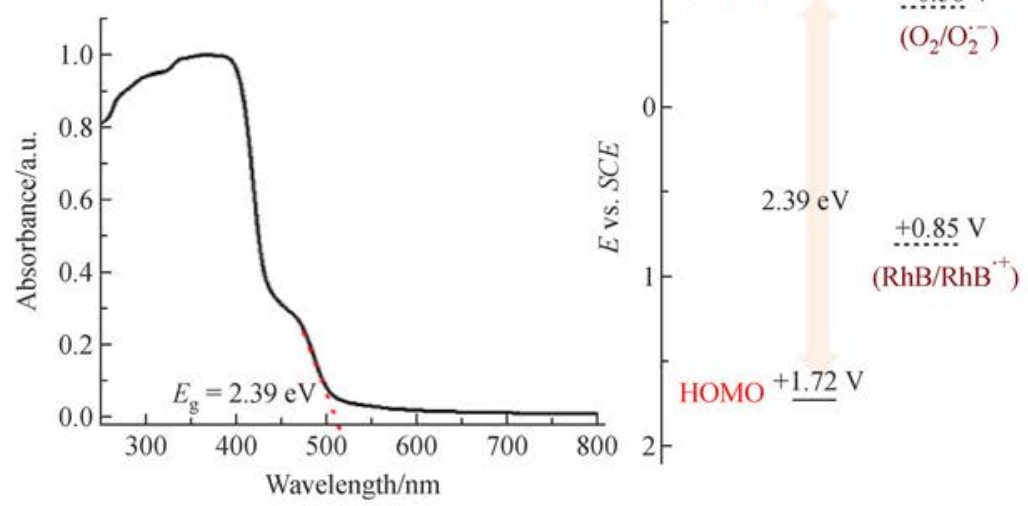

Fig. 1 (a) Structure, (b) HR-TEM, (c) SEM, (d) FTIR, (e) UV-Vis diffuse reflectance (DR) spectra and (f) energy band positions of CTFTh@SBA15.

Table 1 Physical properties of the CTF and reference materials

\begin{tabular}{lccc}
\hline Sample & $S_{\mathrm{BET}} /\left(\mathrm{m}^{2} \cdot \mathrm{g}^{-1}\right)$ & Pore volume $/\left(\mathrm{cm}^{3} \cdot \mathrm{g}^{-1}\right)$ & Average pore size $/ \mathrm{nm}$ \\
\hline SBA-15 & 596 & 0.91 & 5.4 \\
CTF-Th & 57 & 0.28 & 19.3 \\
CTF-Th@SBA-15 & 374 & 0.59 & 4.1 \\
\hline
\end{tabular}

measured. The wettability of the pure CTF-Th and pure SBA-15 has been also evaluated as references. The images are shown in Fig. S4 (cf. ESM). It was found that water had a contact angle of $24^{\circ}$ on the surface of the hybrid CTFTh@SBA-15, while this contact angle was of $141^{\circ}$ on the pure CTF-Th, of hydrophobic nature. Such a low value $\left(<90^{\circ}\right)$ based on the reported literature [19] confirms the gained hydrophilicity of the CTF-Th@SBA-15 due to the presence of SBA-15 and explains the excellent dispersibility into water.

\subsection{Photocatalytic performance}

The as-prepared CTF-Th@SBA-15 was used for the photodegradation of $\mathrm{RhB}$ in aqueous medium and under visible light illumination $\left(\lambda=460 \mathrm{~nm}, 0.16 \mathrm{~W} \cdot \mathrm{cm}^{-2}\right)$. Figure 2(a) shows the degradation results of RhB using the CTF-Th@SBA-15 as photocatalyst. The concentration of
$\mathrm{RhB}$ decreased sharply within $8 \mathrm{~min}$ of reaction and gradually reached zero after $16 \mathrm{~min}$ of reaction. This observation is with accordance to the photographs of $\mathrm{RhB}$ solutions before and after $16 \mathrm{~min}$ of light irradiation (Fig. S5, cf. ESM). The dye solution, although in a high concentration $\left(50 \mathrm{mg} \cdot \mathrm{L}^{-1}\right)$, became completely colorless within 16 min, which means that the RhB molecules were totally degraded. Thus, CTF-Th@SBA-15 was found to be an excellent catalyst for photodegradation of $\mathrm{RhB}$ under visible light illumination. For quantitatively determining the photodegradation rate of $\mathrm{RhB}$, the $\ln \left(C / C_{0}\right)$ vs. time plot (Fig. 2(a) inset) was also obtained according to the UV-Vis absorption intensity at $570 \mathrm{~nm}$. The degradation process conforms to a first order kinetic equation since the $\ln \left(C / C_{0}\right)$ vs. time plot was linear with a high photodegradation rate of $\mathrm{RhB}\left(0.3651 \mathrm{~min}^{-1}\right)$. In contrast, pure CTF-Th, which was used as reference, shows low photodegradation rate of $0.0850 \mathrm{~min}^{-1}$ (Fig. S6). The 

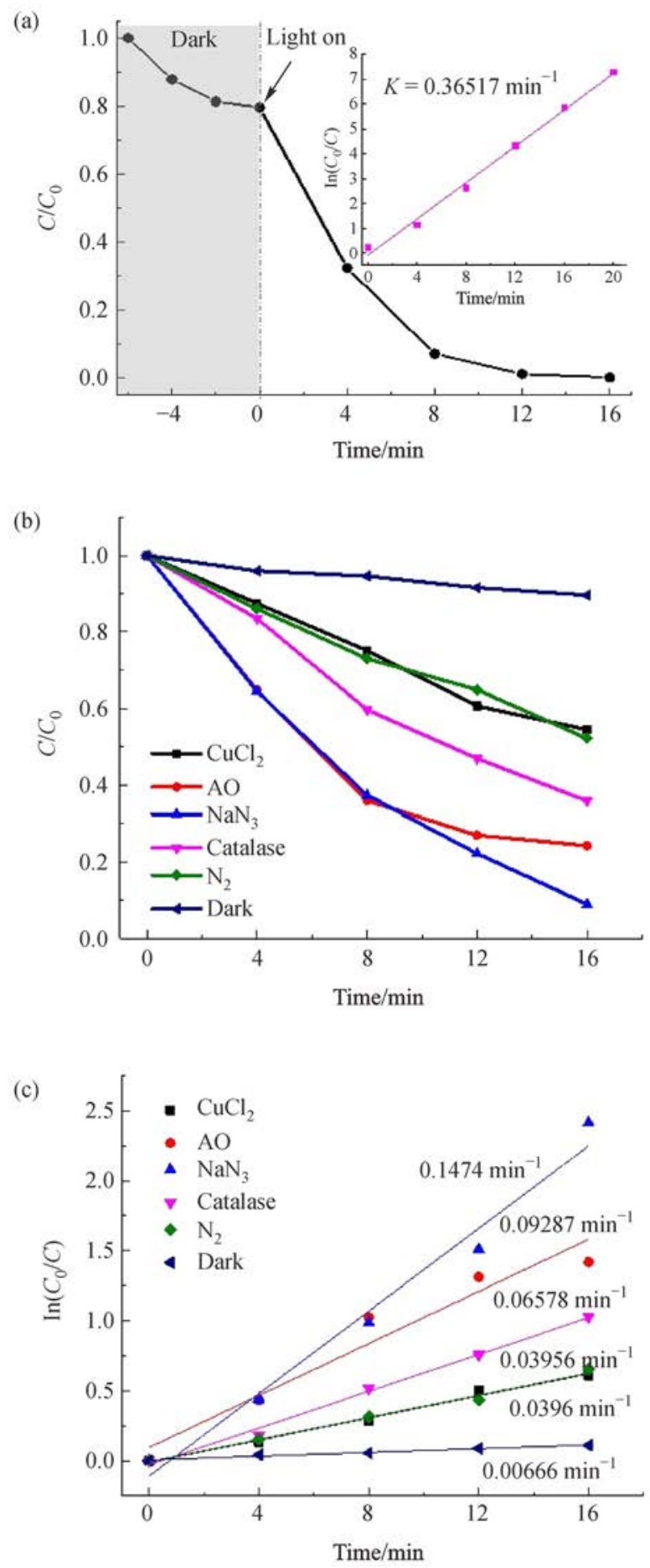

Fig. 2 (a) Visible light-driven degradation of $\mathrm{RhB}$ in water by CTF-Th@SBA15 (Conditions: CTF-Th@SBA15 (3 $\mathrm{mg}), \mathrm{RhB}$ aqueous solution $\left(50 \mathrm{mg} \cdot \mathrm{L}^{-1}, 10 \mathrm{~mL}\right)$, Blue lamp $(\lambda=460 \mathrm{~nm})$ ); (b) Control experiments with different scavengers and (c) Photodegradation rates of the control experiments. $C$ is the concentration of $\mathrm{RhB}$ after light irradiation for a certain period and $C_{0}$ is the concentration of $\mathrm{RhB}$ after reaching adsorption/desorption equilibrium in the dark. enhanced photocatalytic efficiency could be explained by three key points: (i) high BET surface area $\left(374 \mathrm{~m}^{2} \cdot \mathrm{g}^{-1}\right)$ of the hybrid system, which offers a large reactive interface during the catalytic process, (ii) very efficient diffusion of the dye molecules within the mesopores and (iii) excellent adsorption ability in water toward dyes molecules caused by SBA-15 [20].

To investigate the reaction mechanism and reveal the responsible species involved in the reaction process, we conducted a number of control experiments using CTFTh@SBA-15 as photocatalyst. As shown in Figs. 2(b) and 2(c), in the absence of light, almost no degradation of $\mathrm{RhB}$ occurred (rate $=0.0066 \mathrm{~min}^{-1}$ ), whereas under oxygen-free atmosphere, the reaction can still occur but with a very low degradation rate $\left(0.039 \mathrm{~min}^{-1}\right)$. It is important to mention, that in the photodegradation process of organic dyes in water, it is well known that the reactive species such as superoxide $\left(\mathrm{O}_{2}{ }^{-}\right)$, singlet oxygen $\left({ }^{1} \mathrm{O}_{2}\right)$, and the photogenerated hole $\left(\mathrm{h}^{+}\right)$play important roles in the photocatalytic process [21-23]. To underline the contribution of these active species in the RhB degradation using CTFTh@SBA-15, further control experiments were carried out using the following radical scavengers: catalase as $\mathrm{H}_{2} \mathrm{O}_{2}$ scavenger, sodium azide $\left(\mathrm{NaN}_{3}\right)$ as the ${ }^{1} \mathrm{O}_{2}$ scavenger, copper chloride $\left(\mathrm{CuCl}_{2}\right)$ as the $\mathrm{O}_{2}{ }^{-}$scavenger, and ammonium oxalate (AO) as the $\mathrm{h}^{+}$scavenger. Visibly, the addition of copper chloride in the reaction led to an expressive decrease in the photodegradation process of $\mathrm{RhB}$ to $0.0395 \mathrm{~min}^{-1}$ compared to $0.3651 \mathrm{~min}^{-1}$ under normal conditions. This result confirmed the ability of CTF-Th@SBA-15 in forming radical oxygen species by reducing oxygen under visible light irradiation, which was already proven in our previous work [12]. These species are responsible for the degradation of $\mathrm{RhB}$, with the main contribution of superoxide $\left(\mathrm{O}_{2}{ }^{-}\right)$. Additionally, the photocatalytic reaction rates decreased significantly by the addition of catalase as $\mathrm{H}_{2} \mathrm{O}_{2}$ scavenger $\left(0.0658 \mathrm{~min}^{-1}\right)$, Aluminum oxalate as the hole scavenger $\left(0.0929 \mathrm{~min}^{-1}\right)$, sodium azide as singlet oxygen scavenger $\left(0.1474 \mathrm{~min}^{-1}\right)$. Therefore, all elements such as the photogenerated hole, active oxygen species, in-situ generated $\mathrm{H}_{2} \mathrm{O}_{2}$ play a role in the direct photooxidation of $\mathrm{RhB}$ [24]. Based on the observations drawn from the experiments described above, we could suggest the following reaction mechanism as shown in Fig. 3: under light irradiation, the charge separation, that occurs within the hybrid CTF photocatalyst, generates electron-hole pairs. The photogenerated hole oxidizes $\mathrm{RhB}$ molecules, possessing an oxidation potential of $+0.85 \mathrm{~V}$ vs. SCE [25]. In the same time, the electron from the LUMO of the CTF-Th@SBA-15 assisted the reductive activation of molecular oxygen into superoxide $\left(\mathrm{O}_{2}{ }^{-}\right)$, which further react with the oxidized $\mathrm{RhB}$ molecules causing the degradation of $\mathrm{RhB}$ [26]. Note that singlet oxygen $\left({ }^{1} \mathrm{O}_{2}\right)$, originating either from energy transfer or from secondary reactions, also took part in the mechanism [27,28]. 


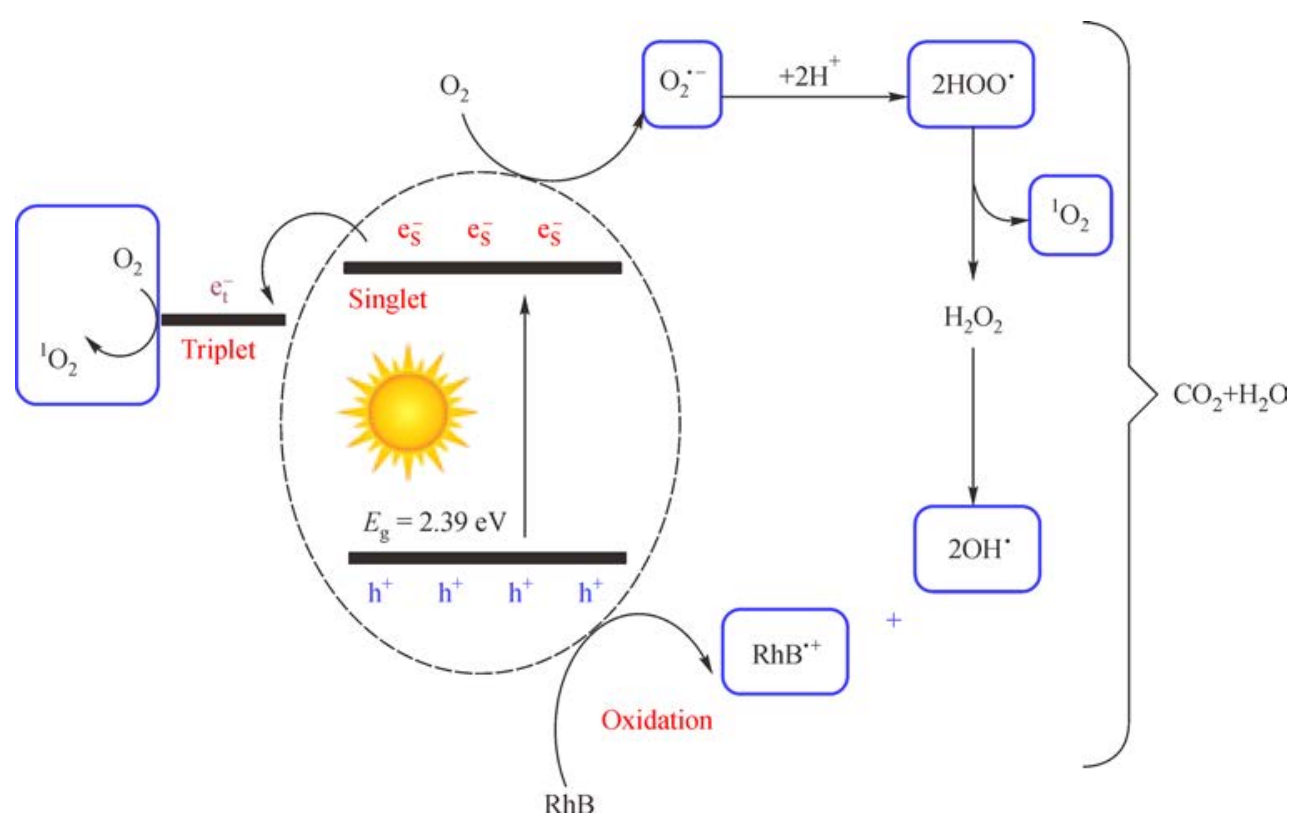

Fig. 3 Suggested reaction mechanism of the photodegradation of RhB using CTF-Th@SBA-15 as photocatalyst.

As we have mentioned previously, the efficient photocatalytic activity of CTF-Th@SBA-15 could be partly attributed to the adsorptive power of SBA-15 present in the photocatalytic system. In order to prove it, we carried out the photodegradation of $\mathrm{RhB}$ in solid-state rather that in aqueous medium (Section 2). As shown in the photographs in Fig. 4(a), the RhB molecules adsorbed clearly on the surface of the CTF-Th@SBA-15, in which the color turned to dark pink. The dye-adsorbed photocatalyst was then subjected to blue light irradiation and the results are presented in Fig. S7(a) (cf. ESM). The UV-Vis peak at around $570 \mathrm{~nm}$ was very strong for the RhB solution without photodegradation. However, the peak was sharply attenuated after $16 \mathrm{~min}$ of reaction and disappeared after 28 min of illumination. Its concentration gradually reached zero with a rate of $0.1197 \mathrm{~min}^{-1}$ (Figs. 4(b) and 4(c)). Chen et al. [20] explained this result by a possible establishment of a strong hydrogen bond interaction between a large number of active hydroxyls on the surface of mesoporous silica and the nitrogen atoms or carbonyl in RhB dye molecules. Note that the degradation rate of $\mathrm{RhB}$ over CTF-Th@SBA-15 in solid state was three times lower than in water $\left(0.3651 \mathrm{~min}^{-1}\right)$. This might be due to the better diffusion the dye molecules into the mesopores in aqueous solution. To further confirm the high adsorption properties of the hybrid photocatalytic system, two more dyes such as $\mathrm{MB}$ and $\mathrm{OrG}$ were tested and subjected to light irradiation. Samely, the catalyst's color, initially yellow, turned to green in case of $\mathrm{MB}$ and orange in case of $\mathrm{OrG}$ as shown in Fig. 4(a), testifying the good adsorption of the dye molecules on the surface and thus confirming the result described above. The concentration of MB decreased gradually and reached almost zero within $80 \mathrm{~min}$, with a very low degradation rate of $0.0301 \mathrm{~min}^{-1}$. In contrast, the OrG photodegradation was faster $\left(0.1322 \mathrm{~min}^{-1}\right.$, cf. Figs. S7(b,c) of ESM).

\section{Conclusions}

In conclusion, a thiophene-containing covalent traizine framework (CTF-Th) on a mesoporous silica SBA-15 was prepared via cyclization polymerization of dicyanide monomer and under $\mathrm{TfOH}$ vapor in a solid state synthesis. The as-prepared CTF-Th@SBA-15 was found to be an efficient, visible-light-active photocatalyst for complete dyes removal both in solvent and dry state. The presence of mesoporous silica SBA-15 played a key role in the rapid degradation of $\mathrm{RhB}$, either in aqueous medium or in solid state. A high capacity removal of methylene blue and orange $\mathrm{G}$ over the hybrid photocatalytic system in solid state was also demonstrated. This could likely be due to the high surface area that SBA-15 imparts to the photocatalytic system as well as its high adsorption capacity toward organic dye molecules. We believe that this study could strength the use of CTFs as a highly efficient visible light photocatalyst for a broader application field and particularly the contaminants removal and water treatment.

\footnotetext{
Acknowledgements K. A. I. Z. acknowledges the Max Planck Society for financial support. C. A. is a recipient of a fellowship through funding of the Excellence Initiative (DFG/GSC 266) of the Graduate School of Excellence "MAINZ" (Materials Science in Mainz).
}

Electronic Supplementary Material Supplementary material is available in the online version of this article at https://doi.org/10.1007/s11705-0191884-2 and is accessible for authorized users. 
(a)

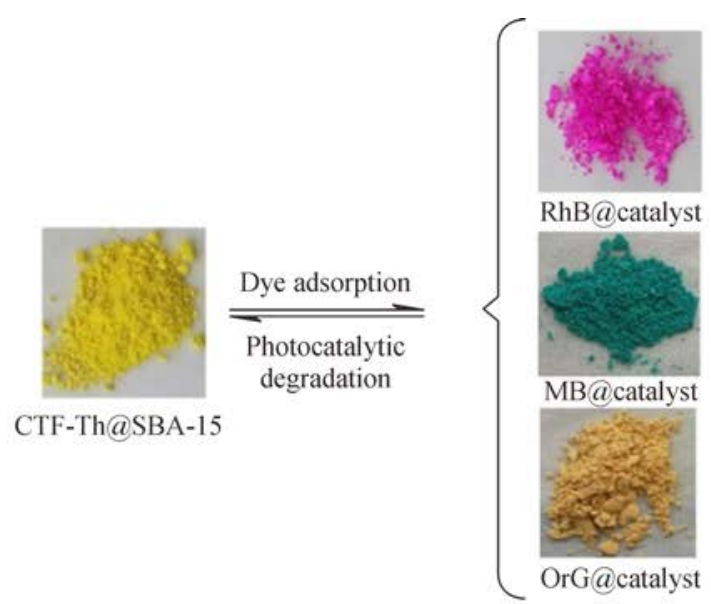

(b)

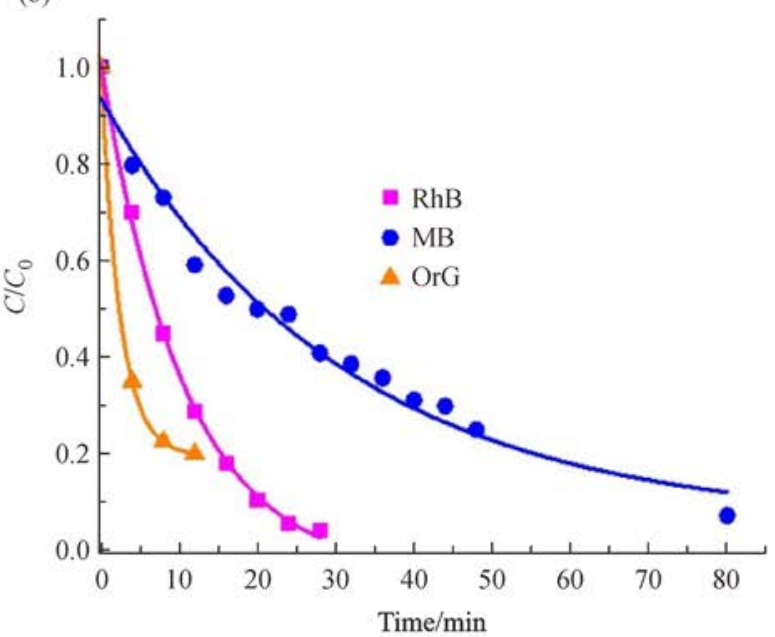

(c)

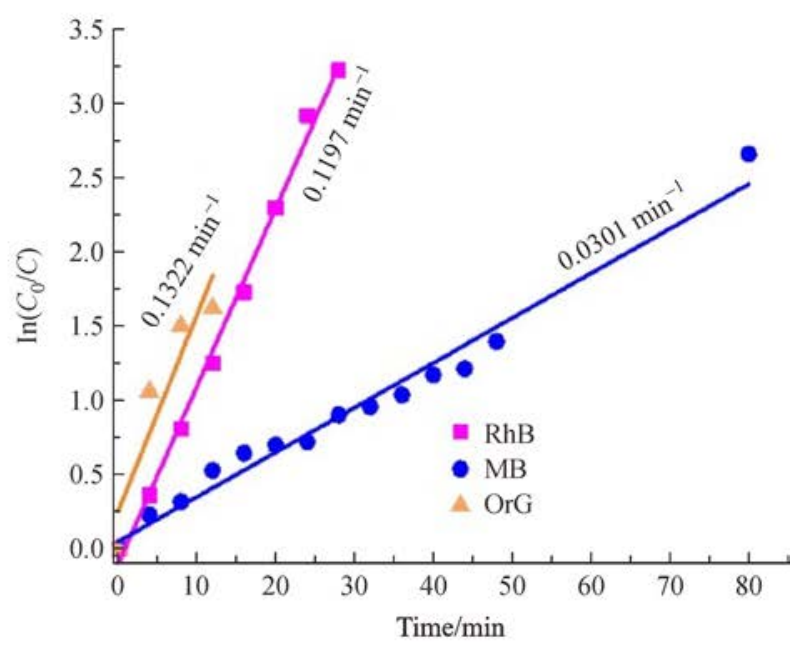

Fig. 4 (a) Photographs of CTF-Th@SBA-15 before and after dye adsorption/degradation, (b) visible light-driven degradation of three different dyes in solid state by CTFTh@SBA-15 and (c) photodegradation rates of the three dyes.
Funding Information Open access funding provided by Projekt DEAL.

Open Access This article is licensed under a Creative Commons Attribution 4.0 International License, which permits use, sharing, adaptation, distribution and reproduction in any medium or format, as long as you give appropriate credit to the original author(s) and the source, provide a link to the Creative Commons licence, and indicate if changes were made. The images or other third party material in this article are included in the article's Creative Commons licence, unless indicated otherwise in a credit line to the material. If material is not included in the article's Creative Commons licence and your intended use is not permitted by statutory regulation or exceeds the permitted use, you will need to obtain permission directly from the copyright holder. To view a copy of this licence, visit http://creativecommons.org/licenses/by/4.0/.

\section{References}

1. Zhang Y, Jin S. Recent advancements in the synthesis of covalent triazine frameworks for energy and environmental applications. Polymers, 2018, 11(1): 31

2. Artz J. Covalent triazine-based frameworks - tailor-made catalysts and catalyst supports for molecular and nanoparticulate species. ChemCatChem, 2018, 10(8): 1753-1771

3. Liu M, Guo L, Jin S, Tan B. Covalent triazine frameworks: Synthesis and applications. Journal of Materials Chemistry. A, Materials for Energy and Sustainability, 2019, 7(10): 5153-5172

4. Zhu X, Tian C C, Mahurin S M, Chai S H, Wang C M, Brown S, Veith G M, Luo H M, Liu H L, Dai S. A superacid-catalyzed synthesis of porous membranes based on triazine frameworks for $\mathrm{CO}_{2}$ separation. Journal of the American Chemical Society, 2012, 134(25): 10478-10484

5. Liebl M R, Senker J. Microporous functionalized triazine-based polyimides with high $\mathrm{CO}_{2}$ capture capacity. Chemistry of Materials, 2013, 25(6): 970-980

6. Hao L, Ning J, Luo B, Wang B, Zhang Y, Tang Z, Yang J, Thomas A, Zhi L. Structural evolution of 2D microporous covalent triazinebased framework toward the study of high-performance supercapacitors. Journal of the American Chemical Society, 2015, 137(1): 219-225

7. Xu F, Yang S, Jiang G, Ye Q, Wei B, Wang H. Fluorinated, sulfurrich, covalent triazine frameworks for enhanced confinement of polysulfides in lithium-sulfur batteries. ACS Applied Materials \& Interfaces, 2017, 9(43): 37731-37738

8. Liu J, Lyu P, Zhang Y, Nachtigall P, Xu Y. New layered triazine framework/exfoliated 2D polymer with superior sodium-storage properties. Advanced Materials, 2018, 30(11): 1705401

9. Bi J, Fang W, Li L, Wang J, Liang S, He Y, Liu M, Wu L. Covalent triazine-based frameworks as visible light photocatalysts for the splitting of water. Macromolecular Rapid Communications, 2015, 36(20): 1799-1805

10. Guo L, Niu Y, Xu H, Li Q, Razzaque S, Huang Q, Jin S, Tan B. Engineering heteroatoms with atomic precision in donor-acceptor covalent triazine frameworks to boost photocatalytic hydrogen production. Journal of Materials Chemistry. A, Materials for Energy and Sustainability, 2018, 6(40): 19775-19781 
11. Xie J, Shevlin S A, Ruan Q, Moniz S J A, Liu Y, Liu X, Li Y, Lau C C, Guo Z X, Tang J. Efficient visible light-driven water oxidation and proton reduction by an ordered covalent triazine-based framework. Energy \& Environmental Science, 2018, 11(6): 1617-1624

12. Huang W, Ma B C, Lu H, Li R, Wang L, Landfester K, Zhang K A I. Visible-light-promoted selective oxidation of alcohols using a covalent triazine framework. ACS Catalysis, 2017, 7(8): 54385442

13. Zhu G, Shi S, Liu M, Zhao L, Wang M, Zheng X, Gao J, Xu J. Formation of strong basicity on covalent triazine frameworks as catalysts for the oxidation of methylene compounds. ACS Applied Materials \& Interfaces, 2018, 10(15): 12612-12617

14. Artz J, Mallmann S, Palkovits R. Selective aerobic oxidation of HMF to 2,5-diformylfuran on covalent triazine frameworkssupported Ru catalysts. ChemSusChem, 2015, 8(4): 672-679

15. Streat M, Sweetland L A. Removal of pesticides from water using hypercrosslinked polymer phases: Part 2- sorption studies. Process Safety and Environmental Protection, 1998, 76(2): 127-134

16. Penner N A, Nesterenko P N, Ilyin M M, Tsyurupa M P, Davankov $\mathrm{V}$ A. Investigation of the properties of hypercrosslinked polystyrene as a stationary phase for high-performance liquid chromatography. Chromatographia, 1999, 50(9): 611-620

17. Kuhn $\mathrm{P}$, Krüger $\mathrm{K}$, Thomas A, Antonietti M. "Everything is surface": Tunable polymer organic frameworks with ultrahigh dye sorption capacity. Chemical Communications, 2008, (44): 58155817

18. Huang W, Ma B C, Lu H, Li R, Wang L, Landfester K, Zhang K A I. Visible-light-promoted selective oxidation of alcohols using a covalent triazine framework. ACS Catalysis, 2017, 7(8): 5438-5442

19. Law K Y. Definitions for hydrophilicity, hydrophobicity, and superhydrophobicity: Getting the basics right. Journal of Physical Chemistry Letters, 2014, 5(4): 686-688

20. Chen J, Sheng Y, Song Y, Chang M, Zhang X, Cui L, Meng D, Zhu
H, Shi Z, Zou H. Multimorphology mesoporous silica nanoparticles for dye adsorption and multicolor luminescence applications. ACS Sustainable Chemistry \& Engineering, 2018, 6(3): 3533-3545

21. Li H, Liu Y, Gao X, Fu C, Wang X. Facile synthesis and enhanced visible-light photocatalysis of graphitic carbon nitride composite semiconductors. ChemSusChem, 2015, 8(7): 1189-1196

22. Urakami H, Zhang K, Vilela F. Modification of conjugated microporous poly-benzothiadiazole for photosensitized singlet oxygen generation in water. Chemical Communications, 2013, 49 (23): 2353-2355

23. Byun J, Landfester K, Zhang K A I. Conjugated polymer hydrogel photocatalysts with expandable photoactive sites in water. Chemistry of Materials, 2019, 31(9): 3381-3387

24. Ghosh S, Kouamé N A, Ramos L, Remita S, Dazzi A, DenisetBesseau A, Beaunier P, Goubard F, Aubert P H, Remita H. Conducting polymer nanostructures for photocatalysis under visible light. Nature Materials, 2015, 14(5): 505-511

25. Yu L, Mao Y, Qu L. Simple voltammetric determination of rhodamine B by using the glassy carbon electrode in fruit juice and preserved fruit. Food Analytical Methods, 2013, 6(6): 1665-1670

26. Huang H B, Wang Y, Cai F Y, Jiao W B, Zhang N, Liu C, Cao H L, Lü J. Photodegradation of rhodamine B over biomass-derived activated carbon supported CdS nanomaterials under visible irradiation. Frontiers in Chemistry, 2017, 5(123): 123

27. Mukthar Ali M, Arya Nair J S, Sandhya K Y. Role of reactive oxygen species in the visible light photocatalytic mineralization of rhodamine B dye by P25-carbon dot photocatalyst. Dyes and Pigments, 2019, 163: 274-284

28. Guo F, Wang K, Lu J, Chen J, Dong X, Xia D, Zhang A, Wang Q. Activation of peroxymonosulfate by magnetic carbon supported Prussian blue nanocomposite for the degradation of organic contaminants with singlet oxygen and superoxide radicals. Chemosphere, 2019, 218: 1071-1081 